\title{
Adaptive Multiple-sliding-mode Control of a VTOL UAV Based on the MRPs
}

\author{
Ling $\mathrm{Yu}^{1 *}$ and $\mathrm{Nan} \mathrm{Nan}^{2}$ \\ ${ }^{1}$ Lab Analyst of Network Information Center, Dalian Polytechnic University, \\ Dalian, Liaoning 116000 China \\ ${ }^{2}$ Department of Mechanical and Electrical Engineering, Jining Polytechnic, \\ Jining, Shandong 272037 China \\ *yuling@dlpu.edu.cn
}

\begin{abstract}
An adaptive multiple-sliding-mode control strategy is proposed in this paper for trajectory tracking control of a vertical take-off and landing unmanned aerial vehicle. First, a nonredundant error model of the aircraft is established based on the Modified Rodrigues Parameters. The cascade property is analyzed, based on which the system is divided into position and attitude subsystems, respectively. Then, an adaptive law is designed to estimate the unknown parameters, based on which a multiple-sliding-mode controller is applied to deal with external disturbances. It is proved by Lyapunov theory that the tracking errors can converge to a small neighborhood of the origin point by choosing appropriate control parameters. Simulation results show that the proposed controller enables the VTOL UAV to track a desired trajectory quickly and accurately.
\end{abstract}

Keywords: Vertical take-off and landing unmanned aerial vehicle; Modified Rodrigues Parameters; Sliding Mode Control; Adaptive; Cascade System

\section{Introduction}

In recent years, the researches and applications of vertical take-off and landing unmanned aerial vehicle (VTOL UAV) have been widely concerned, such as unmanned inspection, rescue in disasters and road traffic supervision. The VTOL UAV is a kind of non-holonomic cascade system with complicated restrictions, which do not satisfy the necessary requirement of smooth feedback stabilization from Brockett, only the nonsmooth or time-varying control law could stabilize this kind of systems [1]. Thus, new control methodologies should be explored for this kind of systems.

Many researches focus on the attitude control of the UAVs, however, trajectory tracking control is a more challenging work due to its strong coupling, unknown parameters, external disturbance, etc. In [2-4], backstepping control and feedback linearization are introduced for trajectory tracking problem. However, they cannot deal with the system uncertainties and external disturbances effectively. In [5-6], intelligent control strategies, such as neural networks, fuzzy control are adopted. However, the convergence rate will limit the applications of these methods in practice. In [7-10], sliding model control and model predictive control can increase the control accuracy with system uncertainties. Concerning with the cascade property of the VTOL UAV, hierarchical control strategy is usually applied, the position and attitude controllers are usually designed separately. However, the stability is usually analyzed based on subsystems, while strictly Lyapunov analysis for closed-loop system is important.

From the analysis above, we propose a novel adaptive multiple-sliding-mode control strategy in this paper. The system model is established based on Modified Rodrigues Parameters (MRPs). Then, the trajectory tracking problem is formulated and system error model is established. The system error model is divided into two parts, translational 
subsystem and rotational subsystem, based on which the controller for each subsystem can be designed separately. For the controller, an adaptive law is designed to estimate the unknown parameters, based on which a multiple-sliding-mode controller is applied to deal with external disturbances. At last, according to the Rodrigues formula and the character of the MRPs, it's proved that the tracking errors of the aircraft are bounded through global stability analysis.

This paper is organized as follows. In Section 2, the system model of VTOL UAV is established based on Modified Rodrigues Parameters (MRPs). In Section 3, trajectory tracking problem is described, and the cascade property is analyzed. In Section 4, an adaptive multiple-sliding-mode control methodology is proposed, and stability of closedloop system is analyzed based on cascade theory. Simulation in Section 5 is carried out to show the effectiveness of the proposed method, followed by the Conclusions in Section 6.

\section{System Model}

Define an earth frame, whose x-axis points northward, y-axis eastward and z-axis downward, namely a North-East-Down frame. We consider the VTOL UAV as a rigid body without deformation, and the system model is described as:

$$
\left\{\begin{array}{l}
\dot{\boldsymbol{\xi}}=\boldsymbol{v} \\
m \dot{\boldsymbol{v}}=\boldsymbol{e}_{3} m g-\boldsymbol{R e}_{3} T+\boldsymbol{d}_{1} \\
\dot{\boldsymbol{\sigma}}=G(\boldsymbol{\sigma}) \boldsymbol{\omega} \\
J \dot{\boldsymbol{\omega}}=-\boldsymbol{\omega} \times J \boldsymbol{\omega}+\boldsymbol{\tau}+\boldsymbol{d}_{1}
\end{array}\right.
$$

where $\xi, v \in \mathbf{R}^{3}$ are the position and velocity vectors under the earth frame. $\boldsymbol{\omega} \in \mathbf{R}^{3}$ is the angle velocity of the rigid body. ${ }^{\boldsymbol{d}_{1}}$ and $\boldsymbol{d}_{2}$ are external disturbances acting on the translational and rotational subsystems satisfying assumption $1 . R \in \mathbf{R}^{3 \times 3}$ is attitude transition matrix, $\boldsymbol{e}_{3}=\left[\begin{array}{lll}0 & 0 & 1\end{array}\right]^{\mathrm{T}}$ is a unit vector. $\boldsymbol{\sigma}$ is the MRPs which is defined as $\sigma=\boldsymbol{r} \tan \alpha / 4$ [11]. $\boldsymbol{r}$ and $\alpha$ represent a rotation axis that does not coincide with one of the original coordinate axes and angle about the axis, respectively. $m$ and $J \in \mathbf{R}^{3 \times 3}$ are the mass and inertia matrix of the rigid body, where $J$ is considered to be a diagonal, positive definite matrix. ${ }^{J_{x}},{ }^{J_{y}}, J_{z}$ are moments of inertia about the there coordinate axes. $g$ is the gravity acceleration. $T$ and $\tau \in \mathbf{R}^{3}$ are the throttle and moment on the rigid body. The expressions of $R(\sigma)$ and $G(\sigma)$ are given in as:

$$
\begin{gathered}
\boldsymbol{R}(\boldsymbol{\sigma})=\boldsymbol{I}_{3}-\frac{4\left(1-\boldsymbol{\sigma}^{\mathrm{T}} \boldsymbol{\sigma}\right)}{\left(1+\boldsymbol{\sigma}^{\mathrm{T}} \boldsymbol{\sigma}\right)^{2}} S(\boldsymbol{\sigma})+\frac{8}{\left(1+\boldsymbol{\sigma}^{\mathrm{T}} \boldsymbol{\sigma}\right)^{2}} S(\boldsymbol{\sigma})^{2} \\
G(\boldsymbol{\sigma})=\frac{1}{2}\left(\frac{1-\boldsymbol{\sigma}^{\mathrm{T}} \boldsymbol{\sigma}}{2} \boldsymbol{I}_{3}+S(\boldsymbol{\sigma})+\boldsymbol{\sigma} \boldsymbol{\sigma}^{\mathrm{T}}\right)
\end{gathered}
$$

Assumption 1. the disturbances $\boldsymbol{d}_{1}$ and $\boldsymbol{d}_{2}$ are bounded with $\left\|D_{1}\right\| \leq \eta_{1}$ and $\left\|D_{2}\right\| \leq \eta_{2}$.

\section{Problem Formulation}

The trajectory tracking problem of a VTOL UAV is investigated, and the objective of this research is to design the control thrust $T_{\mathrm{d}}$ and $\tau_{\mathrm{d}}$, such that the VTOL UAV can track a desired trajectory quickly and accurately. The vector $\left[\begin{array}{lll}x_{\mathrm{d}} & \dot{\boldsymbol{x}}_{\mathrm{d}} & \ddot{\boldsymbol{x}}_{\mathrm{d}}\end{array}\right]$ is the desired 
trajectory which contains position, velocity and acceleration and the desired acceleration $\ddot{x}_{\mathrm{d}}$ satisfies the following assumption 2 .

Assumption 2. The desired acceleration is bounded with $\left\|\ddot{x}_{\mathrm{d}}\right\| \leq v$, where $v$ is a positive constant.

Define the system errors as: position error $\tilde{\xi}=\boldsymbol{\xi}-\boldsymbol{x}_{\mathrm{d}}$, velocity error $\tilde{\boldsymbol{v}}=\boldsymbol{v}-\dot{\boldsymbol{x}}_{\mathrm{d}}$, attitude matrix error $\tilde{R}=R R_{\mathrm{d}}^{\mathrm{T}}, \tilde{\sigma}$ and $\tilde{\boldsymbol{\omega}}$ are MRPs and angular velocity error defined as:

$$
\tilde{\sigma}=\sigma \oplus \tilde{\sigma}_{\mathrm{d}}, \tilde{\omega}=\omega-\tilde{R} \omega_{\mathrm{d}}
$$

So we get the error equations of the system:

$$
\begin{gathered}
\sum_{1}:\left\{\begin{array}{l}
\dot{\tilde{\xi}}=\tilde{\boldsymbol{v}} \\
\dot{\tilde{\boldsymbol{v}}}=g \boldsymbol{e}_{3}-\frac{1}{m} \boldsymbol{n}_{\mathrm{d}} T_{\mathrm{d}}-\ddot{\boldsymbol{x}}_{\mathrm{d}}+\frac{1}{m}(I-\tilde{\boldsymbol{R}})\left(\boldsymbol{n}_{\mathrm{d}} T_{\mathrm{d}}\right)+\boldsymbol{d}_{1}
\end{array}\right. \\
\Sigma_{2}:\left\{\begin{array}{l}
\dot{\tilde{\boldsymbol{\sigma}}}=G(\tilde{\boldsymbol{\sigma}}) \tilde{\boldsymbol{\omega}} \\
\dot{\tilde{\boldsymbol{\omega}}}=\boldsymbol{J}^{-1}\left(-\boldsymbol{\omega} \times \boldsymbol{J} \boldsymbol{\omega}+\boldsymbol{\tau}_{\mathrm{d}}+\boldsymbol{d}_{2}\right)-\left(\tilde{\boldsymbol{R}} \dot{\boldsymbol{\omega}}_{\mathrm{d}}-\tilde{\boldsymbol{R}} S(\tilde{\boldsymbol{\omega}}) \boldsymbol{\omega}_{\mathrm{d}}\right)
\end{array}\right.
\end{gathered}
$$

where equations (5) and (6) are the position and velocity subsystems. $\tilde{\sigma}$ and $\sigma_{\mathrm{d}}$ are calculated from $\tilde{\boldsymbol{R}}$ and $\boldsymbol{R}_{\mathrm{d}}$ by (2). $\tilde{\boldsymbol{\omega}}$ and $\boldsymbol{\omega}_{\mathrm{d}}$ are calculated from $\tilde{\boldsymbol{\sigma}}$ and $\boldsymbol{\sigma}_{\mathrm{d}}$. $\left(\tilde{\boldsymbol{R}} \dot{\boldsymbol{\omega}}_{\mathrm{d}}-\tilde{\boldsymbol{R}} S(\tilde{\boldsymbol{\omega}}) \boldsymbol{\omega}_{\mathrm{d}}\right)$ is the derivative of $\tilde{\boldsymbol{R}} \boldsymbol{\omega}_{\mathrm{d}}$ with respect to time ${ }^{[11]}$. $\boldsymbol{n}_{\mathrm{d}}=\boldsymbol{R}_{\mathrm{d}} \boldsymbol{e}_{3}$ is the last row of the desired attitude matrix. $T_{\mathrm{d}}$ and $\tau_{\mathrm{d}}$ are the controllers that converge the system errors to 0 .

If $\tilde{\boldsymbol{\sigma}}=0$, then the coupled term between the two subsystems $\frac{1}{m}(I-\tilde{\boldsymbol{R}})\left(\boldsymbol{n}_{\mathrm{d}} T_{\mathrm{d}}\right)=0$. So we can consider the position error subsystem as a system with the disturbance $\tilde{\sigma}$. The subsystem (5) constitutes a nominal system ${ }^{\sum_{1}^{\prime}}$ and a coupled term ${ }^{g}$ :

$$
\begin{gathered}
\sum_{1}^{\prime}:\left\{\begin{array}{l}
\dot{\tilde{\boldsymbol{\xi}}}=\tilde{\boldsymbol{v}} \\
\dot{\tilde{\boldsymbol{v}}}=g \boldsymbol{e}_{3}-\frac{1}{m} \boldsymbol{n}_{\mathrm{d}} T_{\mathrm{d}}-\ddot{\boldsymbol{x}}_{\mathrm{d}}+\boldsymbol{d}_{1}
\end{array}\right. \\
h=\frac{1}{m}(I-\tilde{\boldsymbol{R}})\left(\boldsymbol{n}_{\mathrm{d}} T_{\mathrm{d}}\right)
\end{gathered}
$$

\section{Controller Design and Stability Analysis}

First, an adaptive multiple-sliding-mode control method is designed to stabilize the translational error subsystem $\Sigma_{1}^{\prime}$ and rotational error subsystem $\Sigma_{2}$. Then the uniformly ultimately bounded property of the position error subsystem is proven by analyzing the property of the coupled term.

\subsection{Controller Design}

STEP1. Controller design of the position error nominal subsystem ${ }^{\Sigma_{1}^{\prime}}$.

The purpose of controller design of the position error nominal subsystem is to acquire the controller $T_{\mathrm{d}}$ and virtual controller $\boldsymbol{n}_{\mathrm{d}}$, which make the states of ${ }^{\prime}$ uniformly ultimately bounded. 
Define two manifolds $s_{1}=\tilde{\boldsymbol{\xi}}, s_{2}=\tilde{\boldsymbol{v}}-\alpha_{1}\left(s_{1}\right)$, where $\alpha_{1}=-k_{1} s_{1}$ is the virtual controller to be solved. Based on the definition of manifold, we acquire $\dot{\alpha}_{1}=-k_{1}\left(s_{2}-k_{1} s_{1}\right) . \hat{m}$ is the estimated mass of the aircraft. The error of mass estimation $\tilde{m}=\hat{m}-m$ satisfies assumption 3 .

Assumption 3. the error of mass estimation is bounded with $\|\tilde{m}\| \leq \varepsilon_{1}$, where ${ }^{\varepsilon_{1}}$ is a positive constant.

We acquire the controller $T_{\mathrm{d}}$ and virtual controller $\boldsymbol{n}_{\mathrm{d}}$ as the following:

$$
\left\{\begin{array}{l}
T_{\mathrm{d}}=\left\|\hat{m}\left(\boldsymbol{e}_{3} g-\ddot{\boldsymbol{x}}_{\mathrm{d}}+k_{1}\left(s_{1}-k_{1} s_{2}\right)+\frac{c \eta_{1} s_{2}}{\left(s_{2}^{\mathrm{T}} s_{2}+\rho^{2}\right)^{1 / 2}}\right)+s_{1}+k_{2} s_{2}\right\| \\
\boldsymbol{n}_{\mathrm{d}}=\left[\hat{m}\left(\boldsymbol{e}_{3} g-\ddot{\boldsymbol{x}}_{\mathrm{d}}+k_{1}\left(s_{1}-k_{1} s_{2}\right)+\frac{c \eta_{1} s_{2}}{\left(s_{2}^{\mathrm{T}} s_{2}+\rho^{2}\right)^{1 / 2}}\right)+s_{1}+k_{2} s_{2}\right] / T_{\mathrm{d}}
\end{array}\right.
$$

where $k_{1}, k_{2}$ are the control parameters, ${ }^{c}$ and ${ }^{\rho}$ determine the neighborhood which the system errors converge to.

The adaptive law is

$$
\dot{\hat{m}}=\gamma s_{2}^{\mathrm{T}}\left(\boldsymbol{e}_{3} g-\ddot{\boldsymbol{x}}_{\mathrm{d}}+k_{1}\left(s_{1}-k_{1} s_{2}\right)+\frac{c \eta_{1} s_{2}}{\left(s_{2}^{\mathrm{T}} s_{2}+\delta^{2}\right)^{1 / 2}}\right)
$$

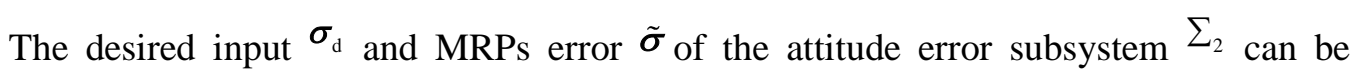
calculated by the virtual controller $\boldsymbol{n}_{\mathrm{d}}$.

Remark 1. Consider that $\omega_{\mathrm{d}}$ and $\dot{\omega}_{\mathrm{d}}$ are required in STEP2, the controller (9) should not contain sign function, so the controller could only converge the trajectory tracking error into a small neighborhood.

STEP2. Controller design of the attitude error subsystem $\sum_{2}$.

The purpose of controller design of the attitude error subsystem is to acquire the controller $\tau_{\mathrm{d}}$, which stabilizes the states of $\sum_{1}^{\prime}$ asymptotically.

Define two manifolds $s_{3}=\tilde{\boldsymbol{\sigma}}, s_{4}=\tilde{\boldsymbol{\omega}}-\alpha_{2}\left(\varepsilon_{3}\right)$, where $\alpha_{1}=-k_{1} s_{1}$ is the virtual controller to be solved. Based on the definition of manifold, we acquire $\dot{\alpha}_{2}=-k_{3} G\left(s_{3}\right)\left(s_{4}-k_{3} s_{3}\right)$.

Define a linear operator ${ }^{L(\cdot)}: \boldsymbol{R}^{3} \rightarrow \boldsymbol{R}^{3 \times 3}$ as

$$
L(\boldsymbol{\mu})=\left[\begin{array}{ccc}
\mu_{1} & 0 & 0 \\
0 & \mu_{2} & 0 \\
0 & 0 & \mu_{3}
\end{array}\right]
$$

So there exists $\boldsymbol{J} \boldsymbol{\mu}=L(\boldsymbol{\mu}) \hat{\Xi}$ for a vector $\boldsymbol{\mu}=\left[\begin{array}{lll}\mu_{1} & \mu_{2} & \mu_{3}\end{array}\right]^{\mathrm{T}}$, where $\Xi=\left[\begin{array}{lll}J_{x} & J_{y} & J_{z}\end{array}\right]^{\mathrm{T}}$. $\hat{\Xi}$ is the estimation of $\Xi$. The error of the estimation $\tilde{\Xi}=\hat{\Xi}-\Xi$ satisfies assumption 4 .

Assumption 4. The estimation error of moments of inertia is bounded with $\|\tilde{\Xi}\| \leq \varepsilon_{2}$, where $\varepsilon_{2}$ is a positive constant.

Hence we get the controller as 
$\tau_{\mathrm{d}}=L(\hat{\Xi})\left(\left(\tilde{\boldsymbol{R}} \dot{\boldsymbol{\omega}}_{\mathrm{d}}-\tilde{\boldsymbol{R}} S\left(\boldsymbol{\varepsilon}_{4}\right) \boldsymbol{\omega}_{\mathrm{d}}\right)+k_{3} G\left(s_{3}\right)\left(s_{4}-k_{3} s_{3}\right)\right)+\boldsymbol{\omega} \times L(\hat{\Xi}) \boldsymbol{\omega}-k_{4} s_{4}-s_{3}-\eta_{2} \operatorname{sgn}\left(s_{4}\right)$

where $k_{3}, k_{4}$ are control parameters.

Remark 2. the operator ${ }^{\operatorname{sgn}(\cdot)}$ of a vector denotes a sign function of each term in the vector.

The adaptive law is

$$
\dot{\hat{\Xi}}=\Gamma^{-1}\left(-S(\boldsymbol{\omega}) L(\boldsymbol{\omega})-L\left(\tilde{\boldsymbol{R}} \dot{\boldsymbol{\omega}}_{\mathrm{d}}-\tilde{\boldsymbol{R}} S\left(s_{4}\right) \boldsymbol{\omega}_{\mathrm{d}}\right)-L\left(k_{3} G\left(s_{3}\right)\left(s_{4}-k_{3} s_{3}\right)\right)\right)^{\mathrm{T}} s_{4}
$$

\subsection{Stability Analysis}

Theorem 1. Consider the position error nominal subsystem ${ }^{\Sigma_{1}^{\prime}}$ of the VTOL UAV, under the control law (9) and adaptive law (10), its position and velocity errors are uniformly ultimately bounded.

Proof. According to the definition of $s_{1}$ and $s_{2}$, manifolds $s_{1}$ and $s_{2}$ are diffeomorphisms of the position error $\tilde{\xi}$ and velocity error $\tilde{\boldsymbol{v}}$, so the stability of $\tilde{\xi}$ and $\tilde{\boldsymbol{v}}$ is equivalent to that of manifolds ${ }^{s_{1}}$ and $s_{2}$.

Define the Lyapunov function

$$
V_{1}=\frac{1}{2} s_{1}^{\mathrm{T}} s_{1}+\frac{m}{2} s_{2}^{\mathrm{T}} s_{2}+\frac{1}{2 \gamma} \tilde{m}^{2}
$$

The derivate of the Lyapunov function is

$$
\dot{V}_{1}=-k_{1} s_{1}^{\mathrm{T}} s_{1}+s_{1}^{\mathrm{T}} s_{2}+s_{2}^{\mathrm{T}}\left(m\left(\boldsymbol{e}_{3} g-\ddot{\boldsymbol{x}}_{\mathrm{d}}+D_{1}+k_{1}\left(s_{2}-k_{1} s_{1}\right)\right)-\boldsymbol{n}_{\mathrm{d}} T_{\mathrm{d}}\right)+\frac{1}{\gamma} \tilde{m} \dot{\hat{m}}
$$

Substitute the control law (9) and adaptive law (10) into (15), we get

$$
\begin{aligned}
\dot{V}_{1}= & -k_{1} s_{1}^{\mathrm{T}} s_{1}+s_{1}^{\mathrm{T}} s_{2}+s_{2}^{\mathrm{T}}\left(m\left(\boldsymbol{e}_{3} g-\ddot{\boldsymbol{x}}_{\mathrm{d}}+D_{1}+k_{1}\left(s_{2}-k_{1} s_{1}\right)\right)\right. \\
& \left.-m\left(\boldsymbol{e}_{3} g-\ddot{\boldsymbol{x}}_{\mathrm{d}}+k_{1}\left(s_{1}-k_{1} s_{2}\right)+\frac{c \eta_{1} s_{2}}{\left(s_{2}^{\mathrm{T}} s_{2}+\delta^{2}\right)^{1 / 2}}\right)-s_{1}-k_{2} s_{2}\right) \\
& +\frac{1}{\gamma} \tilde{m}\left(\dot{\hat{m}}-\gamma s_{2}^{\mathrm{T}}\left(\boldsymbol{e}_{3} g-\ddot{\boldsymbol{x}}_{\mathrm{d}}+k_{1}\left(s_{1}-k_{1} s_{2}\right)+\frac{c \eta_{1} s_{2}}{\left(s_{2}^{\mathrm{T}} s_{2}+\delta^{2}\right)^{1 / 2}}\right)\right) \\
& \leq-k_{1} s_{1}^{\mathrm{T}} s_{1}-k_{2} s_{2}^{\mathrm{T}} s_{2}-m \eta_{1}\left\|s_{2}\right\|\left(\frac{c\left\|s_{2}\right\|}{\left(s_{2}^{\mathrm{T}} s_{2}+\delta^{2}\right)^{1 / 2}}-1\right)
\end{aligned}
$$

when $\left\|s_{2}\right\| \geq \delta /\left(c^{2}-1\right)^{1 / 2}$

$$
\dot{V}_{1} \leq-k_{1} s_{1}^{\mathrm{T}} s_{1}-k_{2} s_{2}^{\mathrm{T}} s_{2}
$$

In can be concluded that ${ }^{S_{1}}$ and $S_{2}$ are uniformly ultimately bounded, so the position and velocity errors $\tilde{\xi}$ and $\tilde{\boldsymbol{v}}$ are uniformly ultimately bounded [12].

Theorem 2. Consider the attitude error subsystem ${ }^{\Sigma_{2}}$ of the VTOL UAV, under the control law (12) and adaptive law (13), the error of MRPs and error of angle velocity are uniformly asymptotically stable. 
Proof. According to the definition of $s_{1}$ and $s_{2}$, manifolds $s_{3}$ and $s_{4}$ are diffeomorphisms of the error of MRPs $\tilde{\sigma}$ and error of angle velocity $\tilde{\boldsymbol{\omega}}$, so the stability of $\tilde{\sigma}$ and $\tilde{\boldsymbol{\omega}}$ is equivalent to that of manifolds $s_{3}$ and $s_{4}$.

Define the Lyapunov function

$$
V_{2}=2 \ln \left(1+s_{3}{ }^{\mathrm{T}} s_{3}\right)+\frac{1}{2} s_{4}{ }^{\mathrm{T}} \boldsymbol{J}_{4}+\frac{1}{2} \tilde{\Xi}^{\mathrm{T}} \Gamma \tilde{\Xi}
$$

Based on the property of MRPs [10]

$$
\sigma^{\mathrm{T}} G(\sigma)=\left(\frac{1+\sigma^{\mathrm{T}} \sigma}{4}\right) \sigma^{\mathrm{T}}
$$

Then we get

$$
\begin{aligned}
& \dot{V}_{2}=-k_{3} s_{3}^{\mathrm{T}} s_{3}+s_{3}^{\mathrm{T}} s_{4}+s_{4}^{\mathrm{T}} \dot{J}_{4}+\tilde{\Xi}^{\mathrm{T}} \Gamma \dot{\hat{\Xi}} \\
& =-k_{3} s_{3}^{\mathrm{T}} s_{3}+s_{3}^{\mathrm{T}} s_{4}+\tilde{\Xi}^{\mathrm{T}} \Gamma \dot{\hat{\Xi}}+s_{4}^{\mathrm{T}}\left(\left(-\boldsymbol{\omega} \times \boldsymbol{J} \boldsymbol{\omega}+\boldsymbol{\tau}_{\mathrm{d}}+D_{2}\right)\right. \\
& \left.-\boldsymbol{J} \cdot\left(\tilde{\boldsymbol{R}} \dot{\boldsymbol{\omega}}_{\mathrm{d}}-\tilde{\boldsymbol{R}} S\left(s_{4}\right) \boldsymbol{\omega}_{\mathrm{d}}\right)-k_{3} \boldsymbol{J} \cdot G\left(s_{3}\right)\left(s_{4}-k_{3} s_{3}\right)\right) \\
& \leq-k_{3} s_{3}^{\mathrm{T}} s_{3}+s_{3}^{\mathrm{T}} s_{4}+s_{4}^{\mathrm{T}}\left(\left(-\boldsymbol{\omega} \times L(\hat{\Xi}) \boldsymbol{\omega}+\boldsymbol{\tau}_{\mathrm{d}}\right)\right. \\
& \left.-L(\hat{\Xi})\left(\tilde{\boldsymbol{R}} \dot{\boldsymbol{\omega}}_{\mathrm{d}}-\tilde{\boldsymbol{R}} S\left(\boldsymbol{\varepsilon}_{4}\right) \boldsymbol{\omega}_{\mathrm{d}}\right)-k_{3} L(\hat{\Xi}) G\left(s_{3}\right)\left(s_{4}-k_{3} s_{3}\right)\right) \\
& +\tilde{\Xi}^{\mathrm{T}} \Gamma\left(\dot{\hat{\Xi}}-\Gamma^{-1}\left(-S(\boldsymbol{\omega}) L(\boldsymbol{\omega})-L\left(\tilde{\boldsymbol{R}} \dot{\boldsymbol{\omega}}_{\mathrm{d}}-\tilde{\boldsymbol{R}} S\left(s_{4}\right) \boldsymbol{\omega}_{\mathrm{d}}\right)\right.\right. \\
& \left.\left.-L\left(k_{3} G\left(s_{3}\right) s_{4}\right)\right)^{\mathrm{T}} s_{4}\right)+\eta_{2}\left|s_{4}\right|
\end{aligned}
$$

Substitute the control law (12) and adaptive law (13) into (20), we get

$$
\dot{V}_{2} \leq-k_{3} s_{3}^{\mathrm{T}} s_{3}-k_{4} s_{4}^{\mathrm{T}} s_{4}
$$

According to the Barbalat lemma, $\lim _{t \rightarrow \infty} s_{3}=\lim _{t \rightarrow \infty} s_{4}=0$, so the states of attitude error subsystem $\Sigma_{2}$ is globally uniformly asymptotically stable [12].

Theorem 3. Consider the original trajectory tracking error subsystem ${ }^{\Sigma_{1}}$ of the VTOL UAV, the control law (9), (12) and adaptive law (10), (13) can make sure the error of MRPs and error of angle velocity uniformly ultimately bounded with appropriate control parameters.

Proof: First, we analyze the property of the coupled term. The Euclidean norm of the coupled term is as follows:

$$
\|h\|=\frac{T_{\mathrm{d}}}{m} \sqrt{\boldsymbol{n}_{\mathrm{d}}^{\mathrm{T}}\left(2 \boldsymbol{I}_{3}-\tilde{\boldsymbol{R}}^{\mathrm{T}}-\tilde{\boldsymbol{R}}\right) \boldsymbol{n}_{\mathrm{d}}}
$$

Assume $\tilde{\boldsymbol{R}}$ is obtained by rotating $\alpha$ around the axis $\boldsymbol{r}$ which is a unit vector. Using the Rodrigues formula we get

$$
\tilde{\boldsymbol{R}}(\alpha, \boldsymbol{r})=\boldsymbol{I}_{3}+\sin \alpha S(\boldsymbol{r})+(1-\cos \alpha) S(\boldsymbol{r})^{2}
$$

Hence we get

$$
\|h\|=\frac{2 T_{\mathrm{d}}}{m}\left|\sin \frac{\alpha}{2}\right| \sqrt{-\boldsymbol{n}_{\mathrm{d}}^{\mathrm{T}} S(\boldsymbol{r})^{2} \boldsymbol{n}_{\mathrm{d}}}
$$


Obviously, $\boldsymbol{n}_{\mathrm{d}}^{\mathrm{T}} S(\boldsymbol{r})^{2} \boldsymbol{n}_{\mathrm{d}}$ is a quadratic form and $\boldsymbol{n}_{\mathrm{d}}^{\mathrm{T}} \boldsymbol{n}_{\mathrm{d}}=1$, hence we can get $\lambda_{\min }\left(S(\boldsymbol{r})^{2}\right) \leq \boldsymbol{n}_{\mathrm{d}}^{\mathrm{T}}\left(S(\boldsymbol{r})^{2}\right) \boldsymbol{n}_{\mathrm{d}} \leq \lambda_{\max }\left(S(\boldsymbol{r})^{2}\right)$. The operators $\lambda_{\max }(\cdot)$ and $\lambda_{\min }(\cdot)$ denote the maximum and minimum eigenvalues of a matrix. As the eigenvalues of $S(\boldsymbol{r})^{2}$ are $\lambda_{1}=0, \lambda_{2,3}=-1$, consequently:

$$
0 \leq\|h\| \leq \frac{2 T_{\mathrm{d}}}{m}\left|\sin \frac{\alpha}{2}\right|
$$

Since $s_{1}-k_{1} s_{2}=\tilde{v}$, according to (10) and the assumptions 2 and 3

$$
T_{\mathrm{d}} \leq \hat{m}\left(g+v+v+\frac{c \eta_{1}}{2 \delta}\right)+k\left(\left\|s_{1}\right\|+\left\|s_{2}\right\|\right)
$$

where ${ }^{k=\max \left\{1, k_{2}\right\}}$, then

$$
T_{\mathrm{d}}^{2} \leq 2 \hat{m}^{2}\left(g+v+v+\frac{c \eta_{1}}{2 \delta}\right)^{2}+2 k^{2}\left(\left\|s_{1}\right\|^{2}+\left\|s_{2}\right\|^{2}\right)
$$

Define the generalized error states $z_{1}^{\mathrm{T}}=\left[\begin{array}{ll}s_{1}^{\mathrm{T}} & s_{2}^{\mathrm{T}}\end{array}\right]$, according to the definition of Euclidean norm:

$$
\begin{gathered}
\left\|s_{1}\right\|^{2}+\left\|s_{2}\right\|^{2}=\left\|z_{1}\right\|^{2} \\
T_{\mathrm{d}}^{2} \leq 2 \hat{m}^{2}\left(g+v+v+\frac{c \eta_{1}}{2 \delta}\right)^{2}+2 k^{2}\left\|z_{1}\right\|^{2} \text {, then we get: }
\end{gathered}
$$

Hence, we get $T_{\mathrm{d}}^{2} \leq 2 \hat{m}^{2}\left(g+v+v+\frac{c \eta_{1}}{2 \delta}\right)^{2}+2 k^{2}\left\|z_{1}\right\|^{2}$, then we get:

$$
T_{\mathrm{d}} \leq \sqrt{2} \hat{m}\left(g+v+v+\frac{c \eta_{1}}{2 \delta}\right)+\sqrt{2} k\left\|z_{1}\right\|
$$

From the definition of MRPs

$$
\left|\sin \frac{\alpha}{2}\right|=\frac{\|\tilde{\boldsymbol{\sigma}}\|}{1+\|\tilde{\boldsymbol{\sigma}}\|^{2}} \leq\|\tilde{\boldsymbol{\sigma}}\|=\left\|s_{3}\right\|
$$

According to the assumption 2, we get

$$
\|g\| \leq \frac{\sqrt{2} \hat{m}}{m}\left(g+v+v+\frac{c \eta_{1}}{2 \delta}\right)+\frac{\sqrt{2} k}{m}\left\|z_{1}\right\|
$$

From (30) and (27), we can get

$$
\begin{aligned}
\left\|L_{g} V_{1}(t, x)\right\| & \leq 2 \sqrt{2}\left\|s_{2}\right\|\left(\hat{m}\left(g+v+v+\frac{c \eta_{1}}{2 \delta}\right)+k\left\|z_{1}\right\|\right)\left\|s_{3}\right\| \\
& \leq\left(2 \sqrt{2} \hat{m}\left(g+v+v+\frac{c \eta_{1}}{2 \delta}\right)\left\|s_{1}\right\|+2 \sqrt{2} k\left\|z_{1}\right\|^{2}\right)\left\|s_{3}\right\|
\end{aligned}
$$

Consequently, there exists

$$
\left\|L_{g} V_{1}(t, x)\right\| \leq\left(a_{1}\left\|s_{1}\right\|+a_{2}\left\|z_{1}\right\|^{2}\right)\left\|s_{3}\right\|
$$


where

$$
a_{1}=2 \sqrt{2} \hat{m}\left(g+v+v+\frac{c \eta_{1}}{2 \delta}\right) \text { and } a_{2}=2 \sqrt{2} k .
$$

According to theorem 2, under the control law (12) and adaptive law (13), there exists a $\kappa_{\infty}$ function $\beta\left(\|\tilde{\sigma}\|, t-t_{0}\right)$ which satisfies the inequality as follows:

$$
\left\|s_{3}\left(t, t_{0},\|\tilde{\boldsymbol{\sigma}}\|\right)\right\| \leq \beta\left(\|\tilde{\boldsymbol{\sigma}}\|, t-t_{0}\right)
$$

There exists $t_{1}$ when $t>t_{1}, \beta\left(\|\tilde{\boldsymbol{\sigma}}\|, t-t_{0}\right) \leq \varepsilon$, then we get:

$$
\begin{gathered}
\dot{V}_{1} \leq-k_{1} s_{1}^{\mathrm{T}} s_{1}-k_{2} s_{2}^{\mathrm{T}} s_{2}-m \eta_{1}\left\|s_{2}\right\|\left(\frac{c\left\|s_{2}\right\|}{\left(s_{2}^{\mathrm{T}} s_{2}+\delta^{2}\right)^{1 / 2}}-1\right)+a_{1} \varepsilon\left\|s_{2}\right\|+a_{2} \varepsilon\left\|z_{1}\right\|^{2} \\
\leq-a_{3}\left\|z_{1}\right\|^{2}-m \eta_{1}\left\|s_{2}\right\|\left(\frac{c\left\|s_{2}\right\|}{\left(s_{2}^{\mathrm{T}} s_{2}+\delta^{2}\right)^{1 / 2}}-a_{4}\right)
\end{gathered}
$$

where $a_{3}=\min \left\{k_{1}, k_{2}\right\}-a_{2} \varepsilon$ and $a_{4}=\left(1+\frac{a_{1} \varepsilon}{m \eta_{1}}\right)$.

Since $\left\|z_{1}\right\| \leq a_{4} \delta /\left(c^{2}-a_{4}^{2}\right)^{1 / 2}$, consider that $\left\|s_{2}\right\| \leq\left\|z_{1}\right\|$, so there exists $\left\|s_{2}\right\| \leq a_{4} \delta /\left(c^{2}-a_{4}^{2}\right)^{1 / 2}$, then we get

$$
\dot{V}_{1} \leq-a_{3}\left\|z_{1}\right\|^{2}
$$

The generalized error states $z_{1}$ are uniformly ultimately bounded. From the definition of $z_{1}$, the position and velocity errors of the system are diffeomorphisms of $z_{1}$, so they are uniformly ultimately bounded. The controller will make the trajectory tracking error converge to a compact set by choosing appropriate control parameters.

\section{Simulations and Analysis}

Assume that the disturbance force and moment are

$$
\begin{aligned}
D_{1} & =\left[\begin{array}{l}
\sin (\pi t / 10)+\sin (\pi t / 100) \\
\sin (\pi t / 10)+\sin (\pi t / 100) \\
\sin (\pi t / 10)+\sin (\pi t / 100)
\end{array}\right] \times 10^{-1} N \\
D_{2} & =\left[\begin{array}{l}
\sin (\pi t / 10)+\sin (\pi t / 100) \\
\sin (\pi t / 10)+\sin (\pi t / 100) \\
\sin (\pi t / 10)+\sin (\pi t / 100)
\end{array}\right] \times 10^{-2} N \cdot m
\end{aligned}
$$

Assume that the initial position of the VTOL UAV is $\left[\begin{array}{lll}0.5 & 0.5 & 0\end{array}\right]^{\mathrm{T}}$ (unit: $\mathrm{m}$ ). The trajectory is generated in MATLAB to simulate the process of vertical take-off, hover and landing, and test the effect of the controller proposed in this paper.

Firstly, we simulated the stability control of the VTOL UAV. Assume that the initial position of the aircraft is $\left[\begin{array}{lll}1 & 1 & 0\end{array}\right]^{\mathrm{T}}$ (unit: $\mathrm{m}$ ), the desired position is $\left[\begin{array}{lll}0 & 0 & -5\end{array}\right]^{\mathrm{T}}$ (unit: $\mathrm{m}$ ). Figure 1 and Figure 2 show the stability control effect of the position and attitude subsystems. 
Remark 2. Because we take the North-East-Down frame as the earth frame, the desired altitude $-5 \mathrm{~m}$ means that the VTOL UAV aircraft should take off and stabilize itself at the height of $5 \mathrm{~m}$.
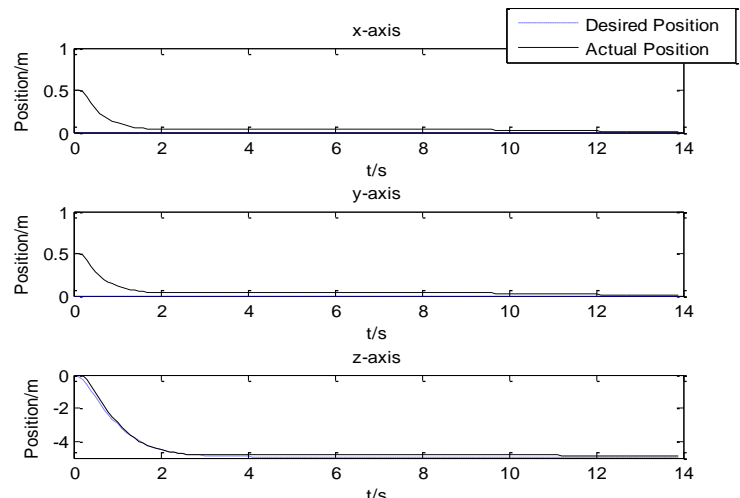

Figure 1. The Stabilization Control Effect of Position Subsystem
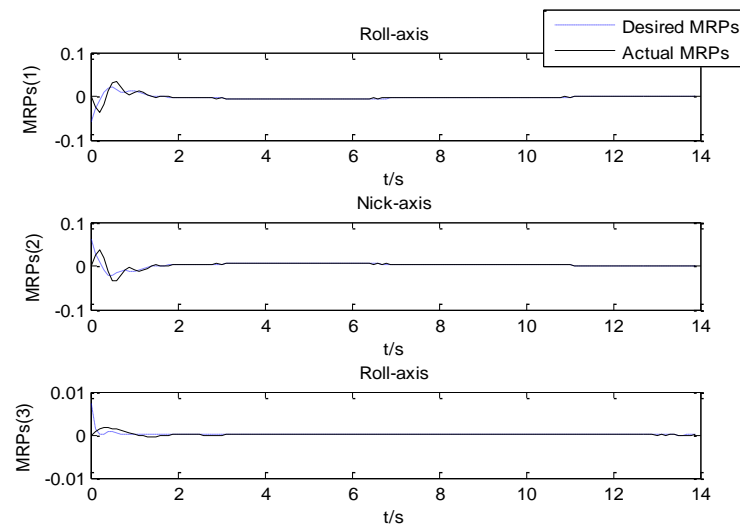

Figure 2. The Stabilization Control Effect of Attitude Subsystem

It is shown in Figure 1 that the controller has a good effect on the stability control of the VTOL UAV. The position error can converge to zero in less than 3 seconds. Figure 2 shows that the MRPs of the system will converge to desired attitude quickly.

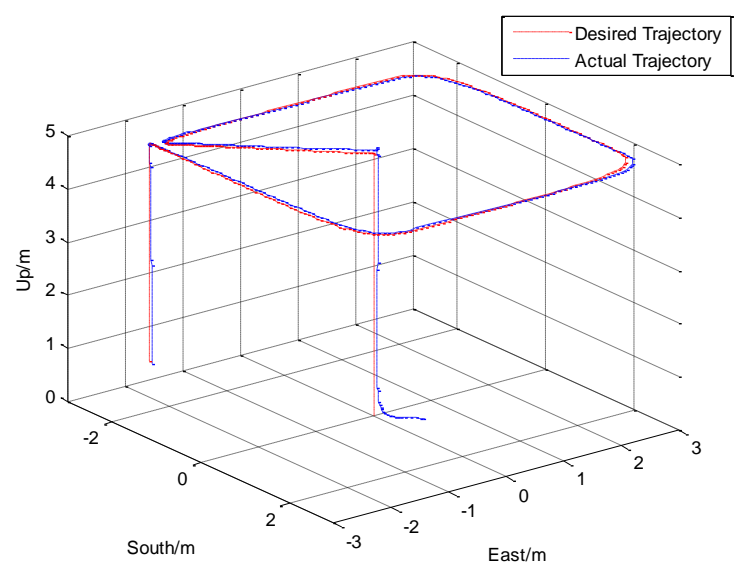

Figure 3. The Trajectory Tracking Effect of the VTOL UAV 

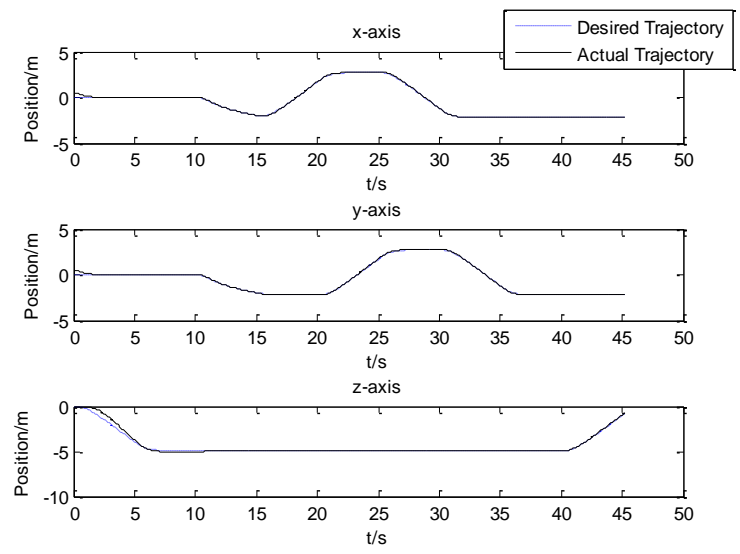

Figure 4. The Control Effect of the Position Subsystem
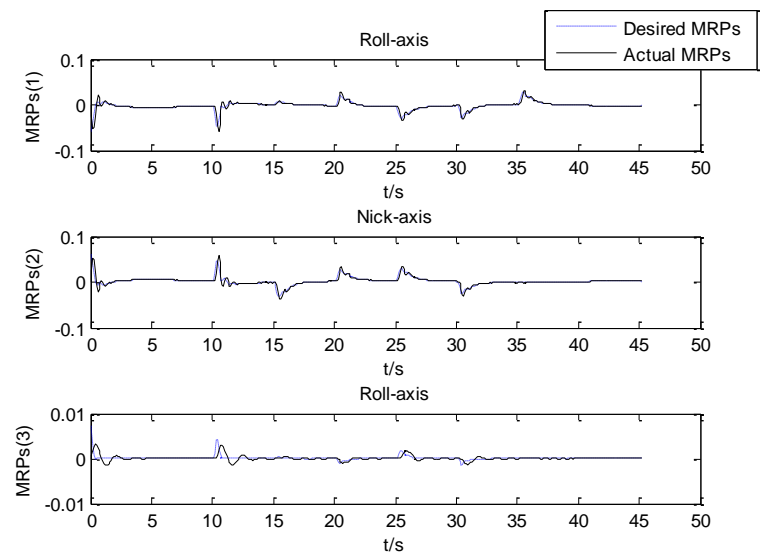

Figure 5. The Control Effect of the Attitude Subsystem

Figure 3 and Figure 4 show that the controller proposed in this paper has an ideal effect of trajectory tracking control, which enables the aircraft tracking an arbitrary trajectory. The trajectory of the aircraft will converge to the desired trajectory quickly even if the system has initial errors. It is shown in Figure 5 that the attitude subsystem has a good tracking effect while the desired MRPs keep changing.

\section{Conclusions}

In this paper, an adaptive based multiple-sliding-mode control methodology is proposed for trajectory tracking problem of a VTOL UAV. The system error model is established based on MRPs. The system error model is divided into two parts, translational subsystem and rotational subsystem, based on which the controller for each subsystem can be designed separately. For the controller, an adaptive law is designed to estimate the unknown parameters, based on which a multiple-sliding-mode controller is applied to deal with external disturbances. Simulations results show that the proposed control strategy can enable the VTOL UAV to track a desired trajectory quickly and accurately. The controller can deal with the uncertainties successfully to increase the control accuracy. 


\section{References}

[1] R. W. Brockett, “Asymptotic stability and feedback stabilization”, Differential Geometric Control Theory, (1983).

[2] Z. Zuo, "Trajectory tracking control design with command-filtered compensation for a quadrotor", IET Control Theory \& Applications, vol. 4, no. 11, (2010).

[3] A. A. Mian and D. B. Wang, "Modeling and backstepping-based nonlinear control strategy for a 6 DOF quadrotor helicopter", Chinese Journal of Aeromautics, vol. 21, no. 3, (2008).

[4] S. Bouabdallsh and R. Siegwart, "Backstepping and slisding-mode techniques applied to an indoor micro quadrotor", In Proceedings of the 2005 IEEE International Conference on Robotics and Automation. Barcelona, Spain, (2005).

[5] M. O. Efe, "Neural network assisted computationally simple control of a quadrotor UAV", IEEE Transactions on Industrial Informatics, vol. 7, no. 2, (2011).

[6] K. M. Zemalache and H. Maaref, "Controlling a drone: Comparison between a based model method and a fuzzy inference system", Applied Soft Computing, vol. 9, no. 2, (2009).

[7] D. Lee, H. J. Kim and S. Sastry, "Feedbcak linearization vs. adaptive sliding mode control for a quadrotor helicopter", International Journal of Control, Automation and Systems, vol. 7, no. 3, (2009).

[8] M. O. Efe, "Battery power loss compensated fractional order sliding mode control of a quadrotor UAV", Asian Journal of Control, vol. 14, no. 2, (2012).

[9] R. Xu and U. Ozguner, "Sliding mode control of a class of underactuated systems", Automatica, vol. 44, no. 1, (2008).

[10] G. V. Raffo, M. G. Ortega and F. R. Rubio, "An integral predictive/nonlinear control structure for a quadrotor helicopter", Automatica, vol. 46, no. 1, (2010).

[11] P. Tsoptras, "Further passivity results for the attitude control problem", IEEE Transactions on Automatic Control, vol. 43, no. 11, (1998).

[12] H. K. Khalil, Nonlinear System, Third Edition, Upper Saddle River, New Jersey: Prentice-Hall, (2002).

\section{Authors}

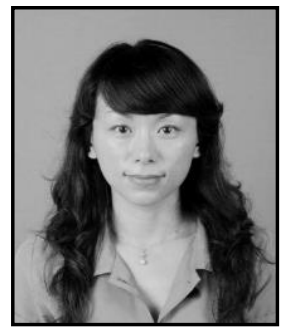

Ling Yu, Received her MS degree in Dalian Polytechnic University. She is now an engineer at Network Information Center of Dalian Polytechnic University and conducts interdisciplinary research and teaching activities on automation, computing science, and network technology.

Nan Nan, Received her MS degree in Shandong University of Science and Technology of China. She is now a lecture at Jining Polytechnic. Her research interests include automatic control, computer aided manufacturing. 
International Journal of Control and Automation Vol.10, No.4 (2017) 